[Article]

\title{
固相反应合成 $\mathrm{LiCoPO}_{4}$ 中间体的反应动力学
}

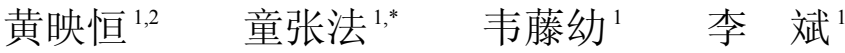 \\ ('广西大学化学化工学院, 南宁 530004; 2桂林理工大学南宁分校冶金工程系, 南宁 530001)
}

\begin{abstract}
摘要: 以磷酸二氢铵、醋酸钴和氢氧化锂为原料, 用低温固相反应合成含 $\mathrm{Li}^{+}$的 $\mathrm{NH}_{4} \mathrm{CoPO}_{4}$ 前驱体, 再经过高温 焙烧合成 $L i C o P O_{4}$ 粉体. 应用傅里叶变换红外光谱 $(F T I R) 、 X$ 射线衍射 $(X R D)$ 和热重-差热分析(TG-DTA)等技术 对合成产物进行表征. 结果表明: 焙烧气氛影响中间体的生成, 含 $\mathrm{Li}^{+}$的前驱体 $\mathrm{NH}_{4} \mathrm{CoPO}_{4}$ 在 $210-500{ }^{\circ} \mathrm{C}$ 的空气 中发生脱水脱氨反应, 制备过程存在“酸碱共同体”的中间体 $\left(\mathrm{CoHPO} \cdot \mathrm{LiCOPO}_{4} \cdot \mathrm{CO}_{2}(\mathrm{OH}) \mathrm{PO}_{4} \cdot \mathrm{Li}_{3} \mathrm{PO}_{4}\right)$. 中间体 生成反应遵循界面反应幂律机理, 表观活化能约 $50.0 \mathrm{~kJ} \cdot \mathrm{mol}^{-1}$, 过程机理函数为 $g(x)=(1-\alpha)^{-1}$. 中间体继续脱 水反应生成 $\mathrm{LiCoPO}_{4}$, 平均表观活化能约为 $54.2 \mathrm{~kJ} \cdot \mathrm{mol}^{-1}$. 物系非晶化和晶化过程对中间体的存在没有直接 的影响, 高温对中间体的分解产物 $\mathrm{LiCoPO}_{4}$ 和 $\mathrm{LiCoPO}_{4}$ 的晶体生长有利, 在 $550{ }^{\circ} \mathrm{C}$ 以上温度中间体可分解得 到完整的 $\mathrm{LiCOPO}_{4}$ 晶体.
\end{abstract}

关键词： $\mathrm{LiCOPO}_{4}$ ；含锂磷酸盐； 中间体；固相反应； 反应机理； 反应动力学 中图分类号: O643; TQ131.1+1

\section{Reaction Kinetics of the Intermediate in Synthesis of $\mathrm{LiCoPO}_{4}$ by Solid-State Reaction}

\author{
HUANG Ying-Heng ${ }^{1,2} \quad$ TONG Zhang-Fa ${ }^{1, *} \quad$ WEI Teng-You ${ }^{1} \quad$ LI Bin $^{1}$ \\ ('School of Chemistry and Chemical Engineering, Guangxi University, Nanning 530004, P. R. China; \\ ${ }^{2}$ Department of Metallurgy Engineering, Guilin University of Technology at Nanning, Nanning 530001, P. R. China)
}

\begin{abstract}
A precursor $\mathrm{NH}_{4} \mathrm{CoPO}_{4}$ containing $\mathrm{Li}^{+}$was synthesized using a low temperature solid-state reaction with ammonium dihydrogen phosphate, cobalt acetate, and lithium hydroxide. $\mathrm{LiCoPO}_{4}$ powder was manufactured by high temperature baking. The products were characterized by Fourier transform infrared (FTIR) spectroscopy, X-ray diffraction (XRD) and thermogravimetry-differential thermal analysis (TG-DTA). The results showed that the formation of the intermediates was effected by the baking atmosphere. $\mathrm{NH}_{4} \mathrm{CoPO}_{4}$ containing $\mathrm{Li}^{+}$was dehydrated and deaminated in air at $210-500{ }^{\circ} \mathrm{C}$ and then the $\left(\mathrm{CoHPO}_{4} \cdot \mathrm{LiCoPO}_{4} \cdot \mathrm{Co}_{2}(\mathrm{OH}) \mathrm{PO}_{4} \cdot \mathrm{Li}_{3} \mathrm{PO}_{4}\right)$ intermediate (acid-base community) was emerged during the reaction process. The intermediate formation reaction mechanism followed the interfacial reaction power-law with an apparent activation energy of $50.0 \mathrm{~kJ} \cdot \mathrm{mol}^{-1}$. The kinetic function was found to be $g(x)=$ $(1-\alpha)^{-1}$. The intermediate was dehydrated to form $\mathrm{LiCoPO}_{4}$ with an average apparent activation energy of $54.2 \mathrm{~kJ} \cdot \mathrm{mol}^{-1}$. The formation of the intermediate was not affected by the process of crystallization or noncrystallization of the materials. High temperatures accelerated the decomposition reaction of the intermediate and then the formation of $\mathrm{LiCoPO}_{4}$ crystals. A perfect crystal of $\mathrm{LiCoPO}_{4}$ was obtained by the decomposition of the intermediate at temperatures higher than $550{ }^{\circ} \mathrm{C}$.
\end{abstract}

Key Words: $\mathrm{LiCoPO}_{4}$; Lithium orthophosphate; Intermediate; Solid state reaction;

Reaction mechanism; Kinetics

Received: November 25, 2010; Revised: February 22, 2011; Published on Web: March 25, 2011.

"Corresponding author. Email: zhftong@sina.com; Tel: +86-771-3233718.

The project was supported by the National Natural Science Foundation of China (20766001), Foundation for Young Scientists of Guangxi, China (0728101), and Research Foundation of Education Bureau of Guangxi, China (200505083).

国家自然科学基金(20766001), 广西青年科学基金(0728101)和广西教育厅科研项目(200505083)资助

(C) Editorial office of Acta Physico-Chimica Sinica 


\section{1 引 言}

在锂离子电池正极材料中, $\mathrm{LiCoPO}_{4}$ 因具有与材 料 $\mathrm{LiMPO}_{4}(\mathrm{M}=\mathrm{Fe}, \mathrm{Ni}, \mathrm{Mn})$ 相近的充放电理论比容 量(约为 $\left.170 \mathrm{mAh} \cdot \mathrm{g}^{-1}\right)^{1,2}$ 被视为高电位锂离子电池 候选正极材料之一, ${ }^{3-5}$ 是锂电池材料研究的热点. 而 且基于 $\mathrm{LiCoPO}_{4}$ 中 $\mathrm{Co}$ 的过渡金属元素的性质, 其作 为磁电材料的应用 ${ }^{6-9}$ 也倍受关注.

$\mathrm{LiCoPO}_{4}$ 常见的制备方法有溶胶-凝胶法和固 相反应合成法. Ehrenberg 等 ${ }^{10,11}$ 应用溶胶-凝胶法制 备含有不同锂离子量的 $\mathrm{Li}_{1-x} \mathrm{CoPO}_{4}$, 研究其电化学 脱锂时晶体结构的变化以及研究不同的制备技术 路线, 特别是两个不同的制备温度 $\left(600\right.$ 和 $\left.800^{\circ} \mathrm{C}\right)$ 对 材料嵌/脱锂行为的影响. ${ }^{10}$ Wolfenstine 等 ${ }^{12}$ 采用固相 反应两步法制备 $\mathrm{LiCoPO}_{4}$, 对材料性质进行了详细 的研究 ${ }^{2,12-14}$, 把 $\mathrm{CoO}$ 和 $\mathrm{LiH}_{2} \mathrm{PO}_{4}$ 混合研磨 $2 \mathrm{~h}$ 后, 在 $375^{\circ} \mathrm{C}$ 下反应 $20 \mathrm{~h}$ 得到前驱体, 将前驱体粉碎、研磨 和造粒, 再在空气或氧气等不同气氛中 $775^{\circ} \mathrm{C}$ 烧结 $48 \mathrm{~h}$, 得到 $\mathrm{LiCoPO}_{4}$. Rissouli 等 ${ }^{4}$ 以 $\left(\mathrm{NH}_{4}\right)_{2} \mathrm{HPO}_{4}$ 和 $\mathrm{Li}_{2} \mathrm{CO}_{3} 、 \mathrm{MCO}_{3}(\mathrm{M}=\mathrm{Mn}, \mathrm{Co})$ 和 $\mathrm{NiO}$, 在 $800{ }^{\circ} \mathrm{C}$ 下焙烧 $48 \mathrm{~h}$, 高温固相反应在制备 $\mathrm{LiMPO}_{4}(\mathrm{M}=\mathrm{Mn}, \mathrm{Co}$, $\mathrm{Ni})$. Grigorova 等 ${ }^{15,16}$ 以 $\mathrm{Li}_{2} \mathrm{CO}_{3} 、 \mathrm{Co}_{3} \mathrm{O}_{4} 、\left(\mathrm{NH}_{4}\right)_{2} \mathrm{HPO}_{4}$ 为 原料, 采用热力学控制的固相反应探讨 $\mathrm{LiCoPO}_{4}$ 粒 度变化的规律. 在 $\mathrm{LiCoPO}_{4}$ 掺杂改性中, 也常采用固 相反应. Han 等 ${ }^{17}$ 采用球磨和微波加热固相反应法 制备 $\mathrm{LiCo}_{1-x} \mathrm{Fe}_{x} \mathrm{PO}_{4} / \mathrm{C}(x$ 为 $0,0.05$ 或 0.1$)$. 本研究组 也曾就 $\mathrm{LiMPO}_{4}(\mathrm{M}=\mathrm{Mn}, \mathrm{Co}, \mathrm{Ni})$ 前驱体固相反应法 制备过程 ${ }^{18-20}$ 进行研究, 发现很难得到高纯度的 $\mathrm{LiMPO}_{4}$ 粉体, 在 $\mathrm{LiMPO}_{4}$ 产品中总存在有一定量的 焦磷酸盐 $\mathrm{M}_{2} \mathrm{P}_{2} \mathrm{O}_{7}$, 这现象与合成过程中存在的 “酸 碱共同体”中间体有关. 因此, 本研究应用 TG-DTA、 XRD、FTIR 等技术探讨前驱体高温固相反应制备 $\mathrm{LiCoPO}_{4}$ 过程中间体反应机理, 为材料进一步的研 究提供理论和实验参考.

\section{2 实 验}

\section{1 材料制备}

分别称取相当于 $0.05 \mathrm{~mol}$ 的 $\mathrm{LiOH} 、 \mathrm{NH}_{4} \mathrm{H}_{2} \mathrm{PO}_{4}$ 和 $\mathrm{Co}\left(\mathrm{CH}_{3} \mathrm{COO}\right)_{2}$ (均为分析纯)试剂, 混合后充分研磨, 滴加几滴作为反应模板剂的 PEG-400(C.P.)和 10-15 滴乙醇水溶液, 使物料成为均匀的粘稠浆料状态. 将反应浆料转入烧杯中, 在 $80^{\circ} \mathrm{C}$ 下恒温反应 $4 \mathrm{~h}$ 得 到固相或粘稠状的含 $\mathrm{Li}^{+}$离子前驱体, 在 $105{ }^{\circ} \mathrm{C}$ 干燥 后, 研磨得到粉体前驱体. 改变 $\mathrm{LiOH}$ 的用量, 可制
备不同 $\mathrm{Li}^{+}$含量的前驱体. 将前驱体直接移至马弗炉 中, 分别在设定的温度下反应 $4 \mathrm{~h}$, 得到不同温度合 成的 $\mathrm{LiCoPO}_{4}$ 产物样品.

\section{2 表征方法}

应用日本理学 $\mathrm{D} / \max 2500$ 型 $\mathrm{X}$ 射线衍射仪对前 驱体样品和产物样品进行表征分析, $2 \theta$ 扫描范围 $5^{\circ}-70^{\circ}$, 扫描速率 5 或 $10\left(^{\circ}\right) \cdot \mathrm{min}^{-1}$; 采用 $\mathrm{Cu}$ 靶, 管电 压 $40 \mathrm{kV}$, 管电流 $200 \mathrm{~mA}$.

称取少量的相同质量的样品置于刚玉坩埚内, 应用德国耐驰 NETZSCH STA 409 PC 热分析仪在 流量为 $100 \mathrm{~mL} \cdot \mathrm{min}^{-1}$ 的空气或氮气中以不同的升 温速率 $\left(5,10,15^{\circ} \mathrm{C} \cdot \mathrm{min}^{-1}\right)$ 升到 $900^{\circ} \mathrm{C}$, 记录升温过 程中质量和热量的变化, 得到 TG-DSC-DTA 曲线.

再分别称取相同质量的样品, 红外光灯照射干 燥后与少量 $\mathrm{KBr}$ 混合, 压成片, 在日本岛津FTIR450 型红外光谱仪上测试红外光谱. 扫描范围为 $400-4000 \mathrm{~cm}^{-1}$, 仪器分辨率为 $4 \mathrm{~cm}^{-1}$.

\section{3 结果与讨论}

\section{1 中间体的组成}

图 1 为不同温度下制备的 $\mathrm{LiCoPO}_{4}$ 体系的 $\mathrm{XRD}$ 谱图. 反应温度较低 $\left(250^{\circ} \mathrm{C}\right)$ 时, $\mathrm{NH}_{4} \mathrm{CoPO}_{4}$ 无 法进一步反应生成 $\mathrm{LiCoPO}_{4}, 400{ }^{\circ} \mathrm{C}$ 以下制备的样 品背底较高, 说明存在较多的非晶态物系, 由化学 方法可检出样品中存在大量的 $\mathrm{Li}^{+}$物系; $450{ }^{\circ} \mathrm{C}$ 以上 制备样品的 XRD 谱图背底较低, 衍射峰较强, XRD 谱图与 $\mathrm{LiCoPO}_{4}$ 标准谱图能基本对称. 特别是 $550{ }^{\circ} \mathrm{C}$ 以上制备的样品 $\mathrm{XRD}$ 谱图背景曲线平整, 并 且衍射峰对称. 即在相同的反应时间内, 随活化温 度升高, 所得样品的 XRD 谱背底越平稳, 峰形对称, 活化温度升高有利于 $\mathrm{LiCoPO}_{4}$ 产物的生成和晶体生

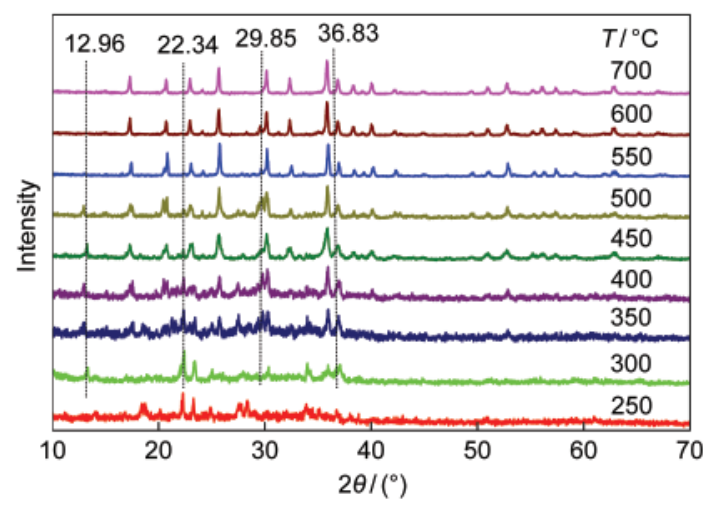

图 1 不同温度下制备的 $\mathrm{LiCoPO}_{4}$ 样品 XRD 谱

Fig.1 XRD patterns of $\mathrm{LiCoPO}_{4}$ synthesized at different temperatures 
长, 有利于非晶态物系转化为晶态物系。

再比较各衍射峰强度变化, 可发现 $2 \theta$ 为 $12.96^{\circ}$ 、 $22.34^{\circ} 、 29.85^{\circ} 、 36.83^{\circ}$ 的衍射峰强度在 $300-500^{\circ} \mathrm{C}$ 间, 随反应温度升高而减少, 这几个衍射峰分别是 $\mathrm{CoHPO}_{4}\left(2 \theta=12.96^{\circ}\right) 、 \mathrm{Li}_{3} \mathrm{PO}_{4}\left(2 \theta=22.34^{\circ}\right)$ 和 $\mathrm{Co}_{2}(\mathrm{OH}) \mathrm{PO}_{4}$ $\left(2 \theta=29.85^{\circ} 、 36.83^{\circ}\right)$ 的特征衍射峰. 其中, $\mathrm{Co}_{2}(\mathrm{OH}) \mathrm{PO}_{4}$ 特征衍射峰和 $\mathrm{LiCoPO}_{4}$ 的特征衍射峰位置很接近, 如 $\mathrm{Co}_{2}(\mathrm{OH}) \mathrm{PO}_{4}$ 特征衍射峰 $\left(2 \theta=36.83^{\circ}\right)$ 强度随温度 升高而减少, 但 $\mathrm{LiCoPO}_{4}$ 的特征衍射峰 $\left(2 \theta=37.06^{\circ}\right)$ 强度随温度升高而增加. 当在 $550^{\circ} \mathrm{C}$ 制备样品时, 所得样品的 XRD 谱在 $2 \theta$ 为 $12.96^{\circ} 、 22.34^{\circ} 、 29.85^{\circ}$ 、 $36.83^{\circ}$ 位置没有发现明显对应的衍射峰.

图 2 是 $270{ }^{\circ} \mathrm{C}$ 制备的中间体样品的 XRD 图. 由 XRD 谱图可定性检测出样品含有 $\mathrm{CoHPO}_{4}$ 、 $\mathrm{LiCoPO}_{4} 、 \mathrm{Co}_{2}(\mathrm{OH}) \mathrm{PO}_{4}$ 和 $\mathrm{Li}_{3} \mathrm{PO}_{4}$ 等晶相的特征峰, 和 图 1中 300-500 ${ }^{\circ} \mathrm{C}$ 样品检测到的物系一样, 包含带 有 $\mathrm{H}^{+}$的 $\mathrm{CoHPO}_{4}$ 和带有 $\mathrm{OH}^{-}$的 $\mathrm{Co}_{2}(\mathrm{OH}) \mathrm{PO}_{4}$, 中间体物 系构成一个“酸碱共同体”, 可用“ $\mathrm{CoHPO}_{4} \cdot \mathrm{LiCoPO}_{4}$ • $\mathrm{Co}_{2}(\mathrm{OH}) \mathrm{PO}_{4} \cdot \mathrm{Li}_{3} \mathrm{PO}_{4}$ ”表示其组成. XRD 谱图 $2 \theta=10^{\circ}$ 附近处有一个较强的衍射峰, 说明材料具有介观结 构特点. 另外 XRD 谱背底较高, 中间体样品中还应 含有非晶态物系.

中间体的存在可能与含 $\mathrm{Li}^{+}$前驱体 $\mathrm{NH}_{4} \mathrm{CoPO}_{4}$ 的 热分解反应过程有关, 含 $\mathrm{Li}^{+}$前驱体 $\mathrm{NH}_{4} \mathrm{CoPO}_{4}$ 含有 $\mathrm{NH}_{4} \mathrm{H}_{2} \mathrm{PO}_{4} 、 \mathrm{Li}_{3} \mathrm{PO}_{4}$ 和 $\left(\mathrm{CH}_{3} \mathrm{COO}\right)_{2} \mathrm{Co}$ 等物质. ${ }^{20}$ 热分解 反应时: 有 $\mathrm{CH}_{3} \mathrm{COOH}$ 酸性气体逸出, 造成反应体系 局部碱性生成 $\mathrm{Co}_{2}(\mathrm{OH}) \mathrm{PO}_{4} ; \mathrm{NH}_{4} \mathrm{CoPO}_{4}$ 脱氨反应生 成 $\mathrm{CoHPO}_{4}$. 固相中 $\mathrm{CoHPO}_{4}$ 和 $\mathrm{Co}_{2}(\mathrm{OH}) \mathrm{PO}_{4}$ 物质扩散 速率比较慢, 没有能进一步瞬时反应生成其它物质.

图 3 为不同温度下制备的 $\mathrm{LiCoPO}_{4}$ 和前驱体样

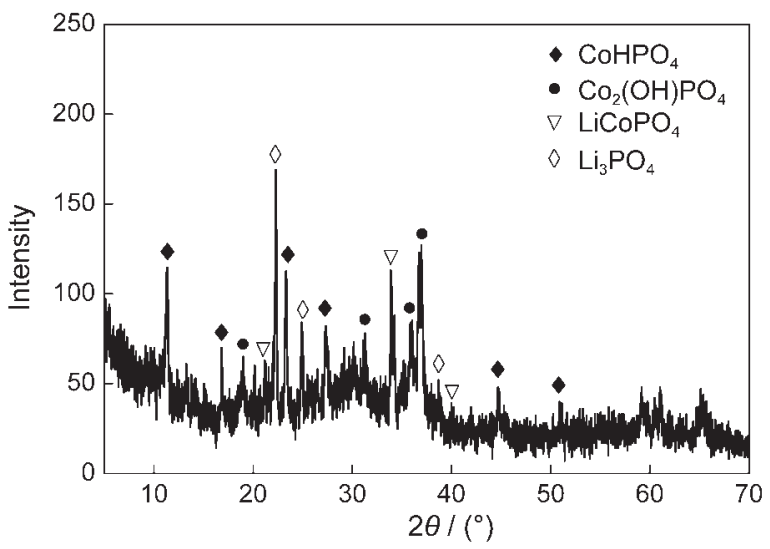

图 2 在 $270{ }^{\circ} \mathrm{C}$ 合成的中间体 XRD 图

Fig.2 XRD patterns of intermediate synthesized at $270{ }^{\circ} \mathrm{C}$

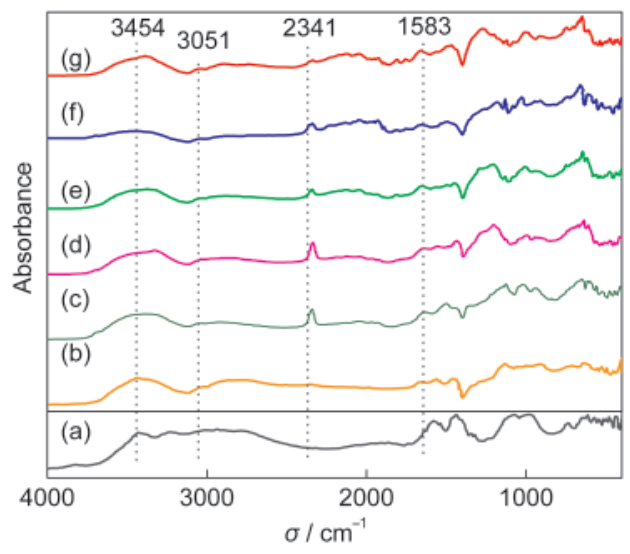

图 3 前驱体和不同温度下制备 $\mathrm{LiCoPO}_{4}$ 样品的 FTIR 光谱

Fig.3 FTIR spectra of precursor and $\mathrm{LiCoPO}_{4}$ synthesized at different temperatures

(a) precursor, (b) $250{ }^{\circ} \mathrm{C}$, (c) $300{ }^{\circ} \mathrm{C}$, (d) $400{ }^{\circ} \mathrm{C}$, (e) $500{ }^{\circ} \mathrm{C}$, (f) $600{ }^{\circ} \mathrm{C},(\mathrm{g}) 700{ }^{\circ} \mathrm{C}$

品的红外吸收光谱. 前驱体在 3256-2786 $\mathrm{cm}^{-1}$ 和 $1448-1474 \mathrm{~cm}^{-1}$ 有 $\mathrm{NH}_{4}^{+}$的特征峰. 在 $250{ }^{\circ} \mathrm{C}$ 制备的 样品这个特征峰的吸收强度下降了, 说明部分前 $\mathrm{NH}_{4} \mathrm{CoPO}_{4}$ 驱体反应生成了产物 $\mathrm{LiCoPO}_{4}$, 此时中间 体含有与前驱体和产物 $\mathrm{LiCoPO}_{4}$ 相似或相同的组 分. 在 $300^{\circ} \mathrm{C}$ 以上制得样品的红外光谱中没有了 $\mathrm{NH}_{4}{ }^{+}$的特征峰. $3051 \mathrm{~cm}^{-1}$ 对应 $\mathrm{OH}^{-}$的吸收光谱的吸 收强度, 在 $300-500{ }^{\circ} \mathrm{C}$ 间, 随温度升高而减少. 另 外, 在 $1000 \mathrm{~cm}^{-1}$ 附近的 $\mathrm{PO}_{4}^{3-}$ 特征峰位置, 也因 $\mathrm{NH}_{4}^{+}$ 和 $\mathrm{Li}^{+}$的电子诱导作用和空间效应的不同而有所不 同..$^{21,22}$

\section{2 影响中间体存在的因素}

\subsection{1 投料量的影响}

图 4 为前驱体和不同 $\mathrm{Li}^{+}$含量的前驱体的 XRD 图, 衍射峰 $2 \theta=10.07^{\circ}$ 与斜方晶系 $\mathrm{NH}_{4} \mathrm{CoPO}_{4}$ 晶体的 图谱(PDF\#21-0793) 特征衍射峰相吻合. 不同 $\mathrm{Li}^{+}$含 量的前驱体样品中物系不同, $n_{\mathrm{Co}}: n_{\mathrm{Li}}=1: 0.2$ 和 $n_{\mathrm{Co}}$ : $n_{\mathrm{Li}}=1: 0.4$ 前驱体样品中组分基本相同, 均为 $\mathrm{NH}_{4} \mathrm{Co}-$ $\mathrm{PO}_{4} 、 \mathrm{NH}_{4} \mathrm{H}_{2} \mathrm{PO}_{4} 、 \mathrm{Co}_{7} \mathrm{H}_{4}\left(\mathrm{PO}_{4}\right)_{6} 、 \mathrm{Co}_{3}\left(\mathrm{PO}_{4}\right)_{2} 、 \mathrm{Li}_{3} \mathrm{PO}_{4}$ 、 $\mathrm{Co}\left(\mathrm{CH}_{3} \mathrm{COO}\right)_{2}$ 等; 含 $\mathrm{Li}^{+}$为 $n_{\mathrm{Co}}: n_{\mathrm{Li}}=1: 0.6$ 的前驱体样品 中主要含有 $\mathrm{NH}_{4} \mathrm{CoPO}_{4} 、 \mathrm{NH}_{4} \mathrm{H}_{2} \mathrm{PO}_{4} 、 \mathrm{Co}_{3}\left(\mathrm{PO}_{4}\right)_{2}$ 、 $\mathrm{Li}_{3} \mathrm{PO}_{4}$ 等. 因为 $\mathrm{LiOH}$ 投料量不同, 前驱体样品中组 分含量不同, 前驱体的 XRD 图也不同, 并且各衍射 峰的积分强度不同.

图 5 为不同 $\mathrm{Li}^{+}$含量前驱体的 DTG 图, DTG 曲 线失重峰数量不随 $\mathrm{LiOH}$ 的投料量变化而变化, 但 随着 $\mathrm{LiOH}$ 投料量的增加, DTG 曲线峰形更加明显, 


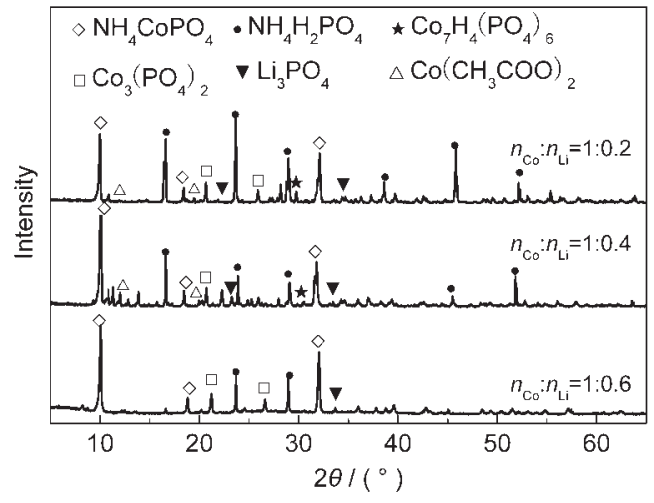

图 4 不同 $\mathrm{Li}^{+}$含量前驱体的 XRD 谱

Fig.4 XRD patterns of precursors containing different $\mathrm{Li}^{+}$quantities

说明 $\mathrm{LiOH}$ 投料量的增加, 提高前驱体的失重速率. 另, 第(1)-(4)组峰随着 $\mathrm{LiOH}$ 投料量的增加, 失重峰 存在向高温方向偏移的现象, 第(5)组峰则向低温方 向偏移. 不同的 $\mathrm{LiOH}$ 投料量改变了前驱体各组分 相对量, 也改变中间体(XRD 谱见图 6)的组分相对 量, 改变了体系中物质颗粒间的相互作用, 改变前 驱体和中间体脱水脱氨的温度.

图 6 为不同 $\mathrm{Li}^{+}$含量的前驱体在 $270{ }^{\circ} \mathrm{C}$ 反应产 物的 XRD 图, 反应的温度选择在第(3)失重之后, 观 测样品的组分和晶态. 样品中存在晶态和非晶态物 系, $\mathrm{LiOH}$ 投料量越大, 谱图背底曲线越高, 非晶态 物系较多; 在 $n_{\mathrm{Co}}: n_{\mathrm{Li}}=1: 0.2$ 的产物中, 主要的晶态物 系为 $\mathrm{NH}_{4} \mathrm{CoPO}_{4} 、 \mathrm{LiCoPO}_{4} 、 \mathrm{LiPO}_{4}$ 等, 但在 $n_{\mathrm{Co}}: n_{\mathrm{Li}}=1$ : 0.6 的产物样品中, 主要的晶态物系为 $\mathrm{NH}_{4} \mathrm{CoPO}_{4}$ 、 $\mathrm{LiCoPO}_{4} 、 \mathrm{LiPO}_{4} 、 \mathrm{Co}_{3}\left(\mathrm{PO}_{4}\right)_{2} 、 \mathrm{CoHPO}_{4} 、 \mathrm{Co}_{11}\left(\mathrm{HPO}_{3}\right)_{8}(\mathrm{OH})_{6}$ 等, 与图 2 在 $270{ }^{\circ} \mathrm{C}$ 合成的中间体的组分相似. 增加 $\mathrm{LiOH}$ 的投料量有利于中间体的生成.

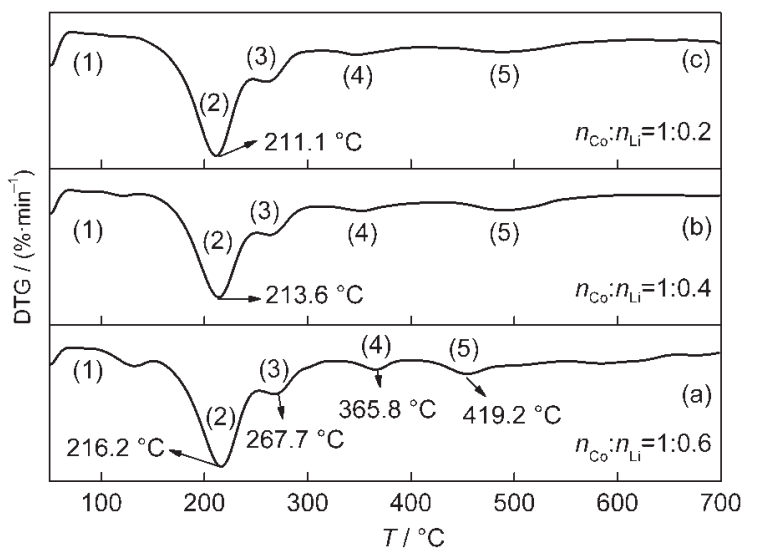

图 5 不同 $\mathrm{Li}^{+}$含量的前驱体的 DTG 图

Fig.5 DTG curves of precursors containing different $\mathbf{L i}^{+}$quantities

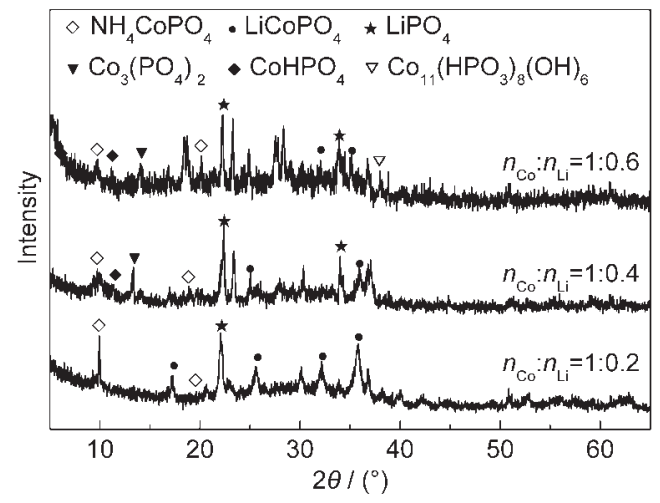

图 6 不同 $\mathrm{Li}^{+}$含量前驱体在 $270^{\circ} \mathrm{C}$ 反应产物的 XRD图

Fig.6 XRD patterns of decomposition products of precursors containing different $\mathrm{Li}^{+}$quantities at $270{ }^{\circ} \mathrm{C}$

图 7 为不同 $\mathrm{Li}^{+}$含量的前驱体在 $500{ }^{\circ} \mathrm{C}$ 反应产 物的 XRD 图, 前驱体的反应产物样品物系主要为 $\mathrm{Co}_{2} \mathrm{P}_{2} \mathrm{O}_{7}$ 和 $\mathrm{LiCoPO}_{4}$. 积分强度最大的衍射峰为: $\mathrm{Co}_{2} \mathrm{P}_{2} \mathrm{O}_{7}$ 晶面(022)特征衍射峰 $2 \theta=29.61^{\circ}$ 和 $\mathrm{LiCoPO}_{4}$ 晶面(131)特征衍射峰 $2 \theta=35.89^{\circ}$. 对谱图进行拟合, 求得含 $\mathrm{Li}^{+}$量 $n_{\mathrm{Co}}: n_{\mathrm{Li}}=1: 0.2, n_{\mathrm{Co}}: n_{\mathrm{Li}}=1: 0.4, n_{\mathrm{Co}}: n_{\mathrm{Li}}=1: 0.6$ 前驱体反应产物样品中, $\mathrm{LiCoPO}_{4}$ 和 $\mathrm{Co}_{2} \mathrm{P}_{2} \mathrm{O}_{7}$ 特征衍 射峰积分强度之比分别为 (160/267), (164/253) 和 $(275 / 321)$, 积分强度之比与物系的相对含量有关, $\mathrm{LiCoPO}_{4}$ 和 $\mathrm{Co}_{2} \mathrm{P}_{2} \mathrm{O}_{7}$ 特征衍射峰积分强度之比随 $\mathrm{LiOH}$ 投料量增加而增加.

\section{2 .2 气氛的影响}

图 8 为前驱体 $\left(n_{\mathrm{Co}}: n_{\mathrm{Li}}=1: 1\right) \mathrm{NH}_{4} \mathrm{CoPO}_{4}$ 在空气中不 同升温速率下的 TG-DTG 图, 各升温速率下 TG-DTG 曲线走势基本相同, DTG 曲线峰顶温度随 升温速率增加而增加. 整个过程存在 5 次主要失重

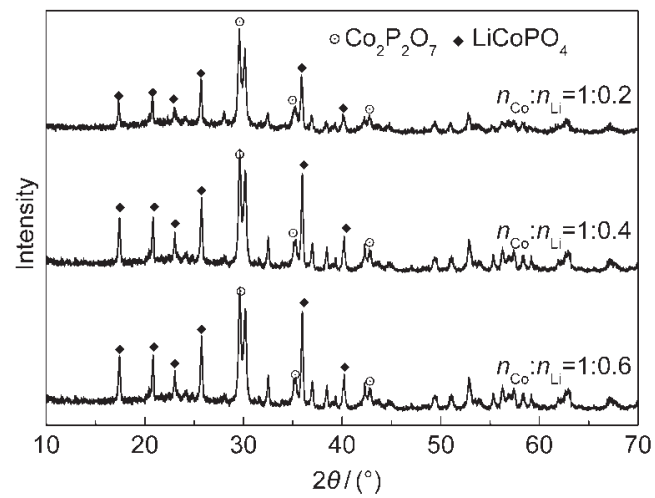

图 7 不同 $\mathbf{L i}^{+}$含量前驱体在 $500{ }^{\circ} \mathrm{C}$ 反应产物的

XRD 图

Fig.7 XRD patterns of decomposition products of precursors containing different $\mathrm{Li}^{+}$quantities at $500{ }^{\circ} \mathrm{C}$ 
现象.

第 1 个失重峰温度范围在 $150-170{ }^{\circ} \mathrm{C}$, 为材料脱 吸附水; 第 2 个失重峰在 $170-250{ }^{\circ} \mathrm{C}$ 之间, 是整个 过程最大失重峰, 为反应物脱水脱氨生成中间体; 第 3 个失重峰在 $270-290{ }^{\circ} \mathrm{C}$ 之间, 为中间体结构调 整, 后继脱水所致; 第 4 个失重峰发生在 $350-379^{\circ} \mathrm{C}$ 之间, 对应中间体脱水生成 $\mathrm{LiCoPO}_{4}$ 的反应; 第 5 个 失重峰在 $530^{\circ} \mathrm{C}$ 以上, 失重很少, 可能是新生成 $\mathrm{LiCoPO}_{4}$ 晶体可能还有结晶水, 进一步脱水并调整 晶体结构. 由 $\mathrm{LiCoPO}_{4}$ 的 TG-DTA 曲线 ${ }^{18}$ 可知, 从 400 至 $700{ }^{\circ} \mathrm{C}$ 之间, $\mathrm{LiCoPO}_{4}$ 对热具有相对稳定性, $\mathrm{LiCoPO}_{4}$ 体系没有明显的失重和吸热或放热峰.

在不考虑反应机理函数的具体形式时, 采用 Ozawa 积分法对图 8 的 TG-DTA 数据进行处理. ${ }^{23,24}$ 根据 Ozawa 方程 ${ }^{25}$ :

$$
\lg \beta=-0.4567 \frac{E}{R T}+\left(\lg \left(\frac{A E}{R}\right)-\lg F(x)-2.315\right)
$$

式中, $F(x)$ 是与转化率有关的函数, $E$ 为活化能 $(\mathrm{kJ}$. $\left.\mathrm{mol}^{-1}\right), R=8.314 \mathrm{~J} \cdot \mathrm{mol}^{-1} \cdot \mathrm{K}^{-1}$ 为气体常数, $A$ 为指前因 子常数 $\left(\mathrm{s}^{-1}\right), T$ 为 $\mathrm{DSC}$ 或 $\mathrm{DTG}$ 峰顶温度 $(\mathrm{K}), \beta$ 为升温 速率 $\left(\mathrm{K} \cdot \mathrm{min}^{-1}\right)$. 在相同的转化率下, $\lg \beta$ 与 $1 / T$ 呈线性 关系, 直线斜率为 $k$, 方程回归系数均在 0.999 以上, 并由 $E=-k R / 0.4567$, 计算过程表观活化能, 结果见 表 1. 根据 Kissinger 方程: $:^{26,27}$

$$
\ln \left(\frac{\beta_{i}}{T_{\mathrm{p}_{i}}^{2}}\right)=\ln \left(\frac{A R}{E}\right)-\frac{E}{R T_{\mathrm{p}_{i}}}, i=1,2,3
$$

利用在不同温度速率 $\beta$ 下记录的 DSC 或 DTG 曲

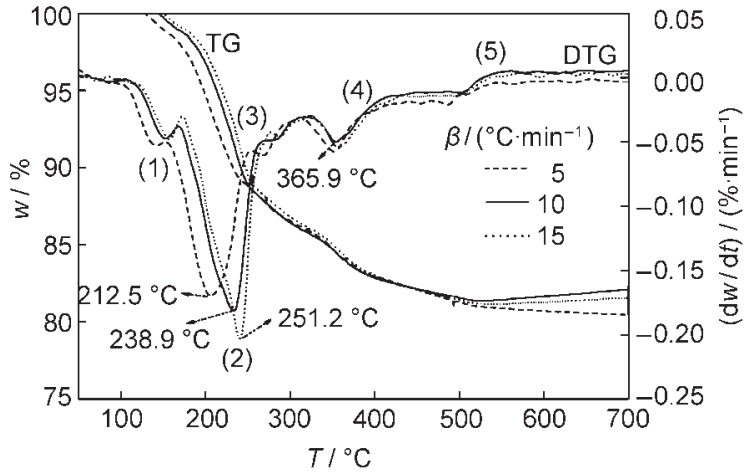

图 8 前驱体 $\left(n_{\mathrm{C}:}: n_{\mathrm{Li}}=1: 1\right)$ 在不同升温速率 $(\beta)$ 下的 TG-DTG图

Fig.8 TG-DTG curves of precursor $\left(n_{\mathrm{C}}: n_{\mathrm{Li}}=1: 1\right)$ at different heating rates $(\beta)$

线, 得到不同的峰顶温度 $T_{\mathrm{p}}$, 由 $\ln \left(\frac{\beta_{i}}{T_{\mathrm{p}_{i}}^{2}}\right)-\frac{1}{T_{\mathrm{p}_{i}}}$ 作图拟合 曲线, 由斜率求 $E$, 由截距求得指前因子的对数 $\ln A$. 具体的计算结果也见表 1 .

图 9 为在氮气氛中的前驱体 $\left(n_{\mathrm{C} 0}: n_{\mathrm{Li}}=1: 1\right)$ 的在 $10{ }^{\circ} \mathrm{C} \cdot \mathrm{min}^{-1}$ 升温速率下的 TG-DTG-DSC 图. 在 $170{ }^{\circ} \mathrm{C}$ 以上, $\mathrm{DTG}$ 曲线存在 2 个明显失重峰, 在 206.3 和 $303.1{ }^{\circ} \mathrm{C}$ 存在了两个分离较好的 DTG 曲线 峰, 之后也不再出现脱水失重峰, 而且各曲线走势 与图 8 明显不同.

在氮气气氛中, 固相表面水蒸气压较小, 有利 于固相反应气体产物从固相中扩散出来, 增大了中 间体的分解速率, 所以在空气气氛 DTG 曲线中原 203 和 $290{ }^{\circ} \mathrm{C}$ 处并没有严格分离的两个热分解峰在

表 1 空气中前驱体 $\left(n_{\mathrm{C} 0}: n_{\mathrm{Li}}=1: 1\right) T G-D T A$ 数据分析结果

\begin{tabular}{|c|c|c|c|c|c|c|c|}
\hline \multirow{2}{*}{ Peak } & \multirow{2}{*}{$\beta /\left({ }^{\circ} \mathrm{C} \cdot \min ^{-1}\right)$} & \multirow{2}{*}{$\begin{array}{c}\text { Peak } \\
\text { temperature } /{ }^{\circ} \mathrm{C}\end{array}$} & \multirow{2}{*}{ Mass loss $/ \%$} & \multirow{2}{*}{ Loss of group } & \multirow{2}{*}{$\frac{\text { Ozawa method }}{E /\left(\mathrm{kJ} \cdot \mathrm{mol}^{-1}\right)}$} & \multicolumn{2}{|c|}{ Kissinger method } \\
\hline & & & & & & $E /\left(\mathrm{kJ} \cdot \mathrm{mol}^{-1}\right)$ & $\ln \left(A / \mathrm{s}^{-1}\right)$ \\
\hline \multirow[t]{3}{*}{ (1) } & 5 & 148.6 & 1.70 & $\mathrm{H}_{2} \mathrm{O}$ & 78.56 & 75.44 & 10.19 \\
\hline & 10 & 161.9 & 1.60 & & & & \\
\hline & 15 & 169.1 & 1.60 & & & & \\
\hline \multirow[t]{3}{*}{ (2) } & 5 & 212.5 & 13.78 & $\mathrm{NH}_{3}+\mathrm{H}_{2} \mathrm{O}$ & 55.26 & 47.72 & 20.14 \\
\hline & 10 & 238.9 & 13.40 & & & & \\
\hline & 15 & 252.1 & 13.30 & & & & \\
\hline \multirow[t]{3}{*}{ (3) } & 5 & 275.6 & - & $\mathrm{H}_{2} \mathrm{O}$ & 127.3 & 124.5 & 25.88 \\
\hline & 10 & 290.9 & - & & & & \\
\hline & 15 & 296.1 & - & & & & \\
\hline \multirow[t]{3}{*}{ (4) } & 5 & 355.6 & 4.58 & $\mathrm{H}_{2} \mathrm{O}$ & 206.9 & 207.0 & 38.45 \\
\hline & 10 & 365.9 & 4.16 & & & & \\
\hline & 15 & 372.7 & 4.00 & & & & \\
\hline \multirow[t]{3}{*}{ (5) } & 5 & 486.4 & - & $\mathrm{H}_{2} \mathrm{O}$ & 132.5 & 116.9 & 16.47 \\
\hline & 10 & 506.0 & - & & & & \\
\hline & 15 & 529.0 & - & & & & \\
\hline
\end{tabular}

Table 1 Data analysis results of TG-DTA of precursor $\left(n_{\mathrm{C} 0}: n_{\mathrm{Li}}=1: 1\right)$ in air 


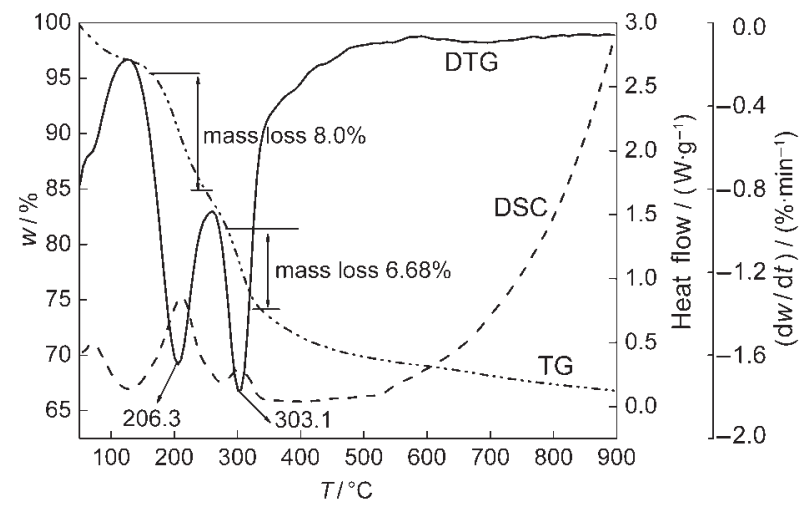

图 9 前驱体 $\left(n_{\mathrm{C} 0}: n_{\mathrm{Li}}=1: 1\right)$ 在氮气中的 TG-DTG-DSC 图

Fig.9 TG-DTG-DSC curves of precursor $\left(n_{\mathrm{C}:}: n_{\mathrm{Li}}=1: 1\right)$ in nitrogen atmosphere

氮气中发生了分离. DSC 曲线也存在两个与 DTG 失 重峰对应的吸热峰, $206.3{ }^{\circ} \mathrm{C}$ 处的吸热量为 $238.2 \mathrm{~J}$. $\mathrm{g}^{-1}$, 对应反应生成中间体 (XRD 谱见图 10)的过程, $303.1{ }^{\circ} \mathrm{C}$ 处的吸热为 $31.56 \mathrm{~J} \cdot \mathrm{g}^{-1}$, 为不稳定的中间体 固相反应脱水生成的 $\mathrm{LiCoPO}_{4}$ 过程.

图 10 是前驱体 $\left(n_{\mathrm{Co}}: n_{\mathrm{Li}}=1: 1\right)$ 在 $270{ }^{\circ} \mathrm{C}$ 氮气中产 物的 XRD 图, 经定性检测, 产物中含有 $\mathrm{LiCoPO}_{4}$. $\mathrm{Co}_{7} \mathrm{H}_{4}\left(\mathrm{PO}_{4}\right)_{6} 、 \mathrm{Li}_{4} \mathrm{P}_{2} \mathrm{O}_{7} 、 \mathrm{NH}_{4} \mathrm{CoPO}_{4} 、 \mathrm{Co}_{3}\left(\mathrm{PO}_{4}\right)_{2}$ 和 $\mathrm{Co}_{2} \mathrm{P}_{2} \mathrm{O}_{7}$ 等, 没有含类似在空气中反应生成的碱性组分, 可 能是在氮气中碱性组分组分更容易脱水生成别的 物质. 由图 9 可知这中间体很快在 $303{ }^{\circ} \mathrm{C}$ 左右脱水 脱氨, 存在的温度没有如在空气中的那么宽.

图 11 为氮气中前驱体 $\left(n_{\mathrm{C} 0}: n_{\mathrm{Li}}=1: 1\right)$ 不同升温速 率的 DTG 图, 在 $170{ }^{\circ} \mathrm{C}$ 以上 2 个主要失重峰随升温 速率增加峰形基本不变, 但峰顶温度和峰高随升温 速率增加而增加, 升温速率 $15^{\circ} \mathrm{C} \cdot \mathrm{min}^{-1}$ 时, DTG 曲 线在 $350{ }^{\circ} \mathrm{C}$ 出现了与在空气气氛中的 $\mathrm{DTG}$ 相似的

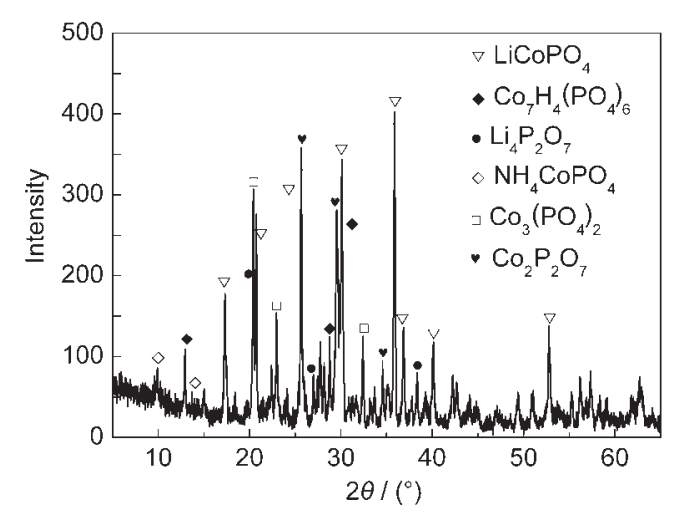

图 10 前驱体 $\left(n_{\mathrm{C} 0}: n_{\mathrm{Li}}=1: 1\right)$ 在 $270{ }^{\circ} \mathrm{C}$ 氮气中反应产物的 XRD图

Fig.10 XRD pattern of product of atmosphere precursor $\left(n_{\mathrm{Co}}: n_{\mathrm{Li}}=1: 1\right)$ in nitrogen atmosphere $\left(270{ }^{\circ} \mathrm{C}\right)$

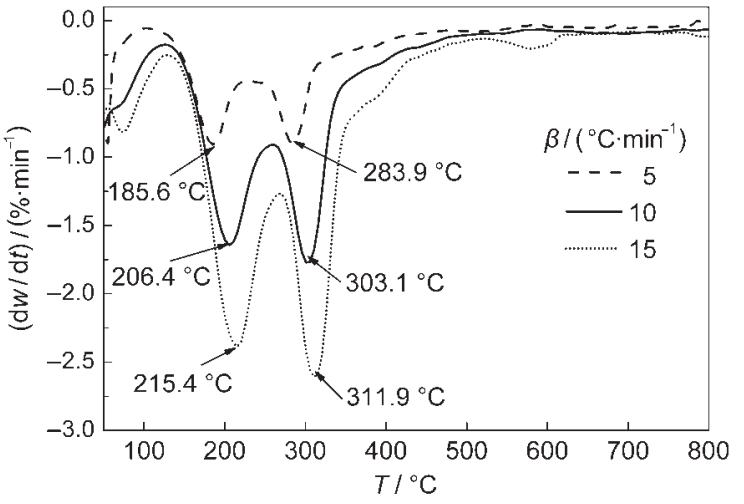

图 11 在氮气中前驱体 $\left(n_{\mathrm{C} o}: n_{\mathrm{Li}}=1: 1\right)$ 不同升温速率的 DTG 图

Fig.11 DTG curves of precursor $\left(n_{\mathrm{C} 0}: n_{\mathrm{Li}}=1: 1\right)$ in nitrogen atmosphere at different heating rates

肩峰, 这类似中间体的存在, 当升温速率较大时, 固 相反应速率增大, 中间产物产生多, 且固相反应气 体产物还未能完全从固相中扩散出来, 中间产物也 还来不及分解成新的产物, 出现了与中间体有关的 分解过程失重峰.

采用 Ozawa 和 Kissinger 方程对峰顶温度和升温 速率进行线性拟合处理, 求得过程活化能和指前因 子见表 2 . 比较表 1 和表 2 数据可知, 在空气气氛中, 前驱体在 $210-250{ }^{\circ} \mathrm{C}$ 存在一个活化能较小的脱水 脱氨反应过程, 后继的几个过程活化能都较高. 在 氮气气氛中, 有 2 个主要反应过程活化能相差不大 也不是很高. 因此, 在氮气气氛中, 反应可以相对顺 畅地进行, 但在空气气氛中, 反应进行到第 2 步时, 后继活化能大, 反应相对缓慢, 是中间体得以生成 的一个必要的条件.

\subsection{3 物系非晶化和晶化过程的影响}

\subsubsection{1 物系非晶化过程对中间体的影响}

活化温度影响了 $\mathrm{LiCoPO}_{4}$ 的生成和晶体生长, 制备过程可认为是前驱体晶态物系首先快速被反 应变为非晶态物系, 非晶态物系再反应转化为晶态 物系 $\mathrm{LiCoPO}_{4}$ 的过程. 这与 $\mathrm{XRD}$ 背景曲线的波动程 度相对应. 由图 1 和图 12 的 XRD 谱, 可应用 Jade 5.0 软件计算XRD 谱线在背景曲线以上面积. 面积与晶 态物系和非晶态物系之间的相对量的有关.

图 13 为 $200-400{ }^{\circ} \mathrm{C}$ 制备的样品的 XRD 谱线 在背景曲线以上面积百分数与温度的关系线. 关系 线显波浪式, 存在 2 个波峰和 2 个波谷, 2 个波峰对 应的温度为 227.3 和 $326.8^{\circ} \mathrm{C}, 2$ 个波谷对应的温度 为 285.9 和 $375.6{ }^{\circ} \mathrm{C}$.

第 1 个波峰的温度 $227.3^{\circ} \mathrm{C}$ 对应前驱体反应 脱氨脱水的温度, 非晶态程度最大. 第 2 个波峰的温 
表 2 氮气中前驱体 $\left(n_{\mathrm{Co}}: n_{\mathrm{Li}}=1: 1\right) T G-D T A$ 数据分析结果

Table 2 Data analysis results of TG-DTA of precursor $\left(n_{\mathrm{C} 0}: n_{\mathrm{Li}}=1: 1\right)$ in nitrogen atmosphere

\begin{tabular}{|c|c|c|c|c|c|c|}
\hline \multirow{2}{*}{ Peak } & \multirow{2}{*}{$\beta /\left({ }^{\circ} \mathrm{C} \cdot \min ^{-1}\right)$} & \multirow{2}{*}{ Peak temperature $/{ }^{\circ} \mathrm{C}$} & \multirow{2}{*}{ Mass loss $/ \%$} & \multicolumn{2}{|c|}{$E /\left(\mathrm{kJ} \cdot \mathrm{mol}^{-1}\right)$} & \multirow{2}{*}{$\frac{\ln \left(A / \mathrm{s}^{-1}\right)}{\text { Kissinger method }}$} \\
\hline & & & & Ozawa method & Kissinger method & \\
\hline \multirow[t]{3}{*}{ (1) } & 5 & 185.58 & 8.04 & 63.89 & 59.33 & 13.76 \\
\hline & 10 & 206.37 & 8.08 & & & \\
\hline & 15 & 215.41 & 8.11 & & & \\
\hline \multirow[t]{3}{*}{ (2) } & 5 & 283.91 & 6.62 & 99.29 & 94.94 & 18.79 \\
\hline & 10 & 303.14 & 6.68 & & & \\
\hline & 15 & 311.96 & 6.63 & & & \\
\hline
\end{tabular}

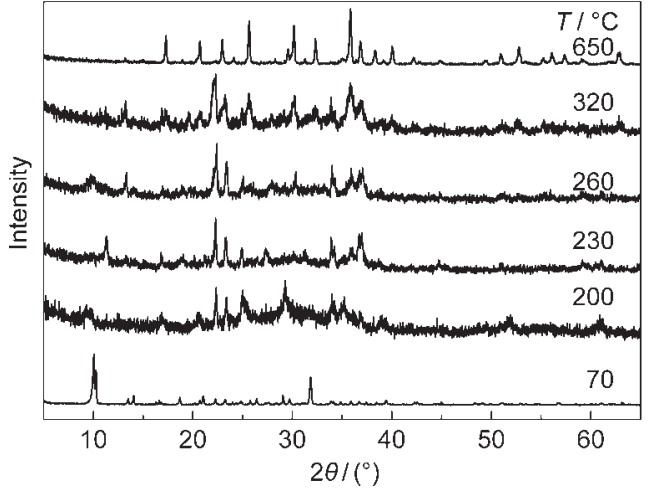

图 12 不同温度下制备的 $\mathrm{LiCOPO}_{4}$ 样品 XRD 谱 Fig.12 XRD patterns of $\mathrm{LiCoPO}_{4}$ synthesized at different temperatures

度 $326.8^{\circ} \mathrm{C}$ 对应中间体反应脱水的温度; 第 1 个波 谷峰的温度 $285.9^{\circ} \mathrm{C}$ 对应中间体生成温度, 非晶态 程度减小. 第 2 个波谷的温度 $375.6{ }^{\circ} \mathrm{C}$ 对应中间体 反应脱水生成 $\mathrm{LiCoPO}_{4}$ 的温度. XRD 谱线在背景曲 线以上面积 $S$ 与温度的关系线基本反映了制备反应 的进度. 应用面积百分数 $S$ 表达非晶化过程进度, 令 $\alpha_{1}=S$, 取 227.3 $-375.6^{\circ} \mathrm{C}$ 间的 $S$ 和 $T$ 值, 将其代入 Coats-Redfern 方程 (式 (3))、Doyle 方程 (式 (4)) 和

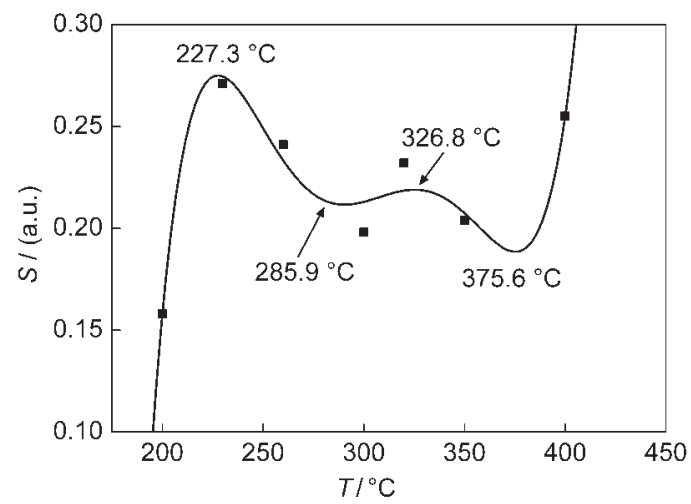

图 $13 X R D$ 谱线在背景曲线以上面积 $(S)$ 与温度 $\left(200-400{ }^{\circ} \mathrm{C}\right)$ 的关系

Fig.13 Relationship between area $(S)$ above the background curve in XRD patterns and temperature $\left(200-400{ }^{\circ} \mathrm{C}\right)$
Achar-Brindley-Slarp-Wendworth 方程 (下面简称 Achar 方程, 式(5)), 选用 15 种常见的固相反应机理 方程进行回归处理, ${ }^{20}$ 拟合系数和标准误差结果见 表3.

$$
\begin{aligned}
& \ln \left(\frac{g(\alpha)}{T^{2}}\right)=\ln \left[\frac{A R}{\beta E}\left(1-\frac{2 R T}{E}\right)\right]-\frac{E}{R T} \\
& \ln g(\alpha)=\ln \left(\frac{A E}{\beta R}\right)-5.3305-1.052 \cdot \frac{E}{R T} \\
& \ln \left[\frac{\mathrm{d} \alpha / \mathrm{d} T}{f(\alpha)}\right]=\ln A-\frac{E}{R T}
\end{aligned}
$$

由表 3 可知, 满足动力学模型拟合系数 $r_{1}>0.99$, 标准误差 $s_{1}<0.2$ 的拟合结果见表中 “加粗斜体”的数 据, 应用 Doyle 方程拟合数据没有一个模型满足最 概然机理拟合条件, 应用积分式(3)和微分式(5)拟合 数据, 满足最概然机理拟合条件是模型 $\mathrm{A} 3$, 即过程机 理 $g(x)=[-\ln (1-\alpha)]^{1 / 3}$, 反应级数 $n=3$, 为集核和核生 长机理. 由 Coats-Redfern方程计算所得的活化能为 $11.37 \mathrm{~kJ} \cdot \mathrm{mol}^{-1}$, 与由 Achar 方程计算所得的活化能 $13.30 \mathrm{~kJ} \cdot \mathrm{mol}^{-1}$ 相差不大, 两者平均值为 $12.33 \mathrm{~kJ}$. $\mathrm{mol}^{-1}$, 比其它反应过程的活化能要小得多.

3.2.3.2 物系晶化过程对中间体的影响

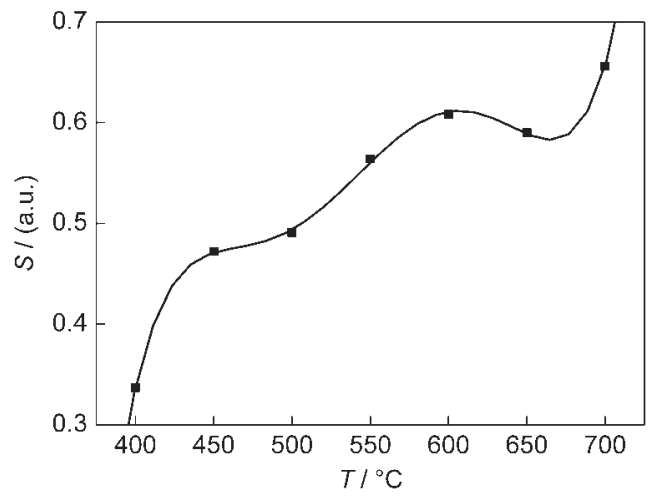

图 14 XRD 谱线在背景曲线以上面积与温度 $\left(400-700{ }^{\circ} \mathrm{C}\right)$ 的关系

Fig.14 Relationship between area above the background curve in XRD patterns and temperature $\left(400-700^{\circ} \mathrm{C}\right)$ 
表 3 物系非晶过程动力学模型拟合系数 $r_{1}$ 及标准误差 $s_{1}$

Table 3 Fitting coefficient $r_{1}$ and standard error $s_{1}$ of kinetic models of amorphous process

\begin{tabular}{|c|c|c|c|c|c|c|c|c|}
\hline \multirow{2}{*}{ Symbol } & \multicolumn{2}{|c|}{ Coats-Redfern equ. } & \multicolumn{2}{|c|}{ Doyle equ. } & \multicolumn{4}{|c|}{ Achar equ. } \\
\hline & $r_{1}$ & $s_{1}$ & $r_{1}$ & $s_{1}$ & $r_{1}$ & $s_{1}$ & $E /\left(\mathrm{kJ} \cdot \mathrm{mol}^{-1}\right)$ & $\ln \left(A / \mathrm{s}^{-1}\right)$ \\
\hline D1 & 0.912 & 0.10 & 0.770 & 0.19 & 0.930 & 0.06 & - & - \\
\hline D2 & 0.913 & 0.19 & 0.771 & 0.20 & 0.629 & 0.11 & - & - \\
\hline D3 & 0.909 & 0.20 & 0.771 & 0.21 & 0.994 & 0.01 & -2.12 & 7.44 \\
\hline $\mathrm{R} 2$ & 0.955 & 0.10 & 0.771 & 0.10 & 0.995 & 0.02 & 9.67 & 3.06 \\
\hline R3 & 0.914 & 0.53 & 0.936 & 0.53 & 0.993 & 0.03 & 9.39 & 2.64 \\
\hline A1 & 0.953 & 0.10 & 0.773 & 0.11 & 0.985 & 0.03 & 8.83 & 3.30 \\
\hline A 1.5 & 0.973 & 0.07 & 0.773 & 0.07 & 0.999 & 0.02 & 10.90 & 4.19 \\
\hline A2 & 0.981 & 0.05 & 0.773 & 0.05 & 0.995 & 0.02 & 11.93 & 4.35 \\
\hline $\mathrm{A} 3$ & 0.991 & 0.03 & 0.773 & 0.04 & 0.990 & 0.04 & 13.30 & 4.39 \\
\hline P1 & 0.959 & 0.99 & 0.770 & 0.09 & 0.999 & 0.01 & 10.51 & 3.80 \\
\hline P2 & 0.984 & 0.04 & 0.770 & 0.05 & 0.989 & 0.04 & 13.21 & 4.43 \\
\hline $\mathrm{P} 3$ & 0.995 & 0.02 & 0.770 & 0.02 & 0.981 & 0.05 & 14.11 & 4.46 \\
\hline P4 & 0.992 & 0.02 & 0.770 & 0.03 & 0.977 & 0.06 & - & - \\
\hline $\mathrm{C} 1$ & 0.998 & 0.01 & 0.790 & 0.01 & 0.965 & 0.08 & - & - \\
\hline $\mathrm{C} 2$ & 0.994 & 0.02 & 0.791 & 0.02 & 0.930 & 0.06 & - & - \\
\hline
\end{tabular}

图 14 为 $400-700^{\circ} \mathrm{C}$ 间制备样品的 XRD 谱线在 背景曲线以上面积与温度的关系线. 关系线显波浪 式, 物系晶化度随温度增加而增加, 同样用 XRD 谱 线在背景曲线以上面积百分数表示晶化过程进度 $\alpha_{2}=S$, 并代入式(3-5), 选用不同的反应机理函数进 行最小二乘法拟合, 拟合系数和标准误差结果见表 4.

由表 4 数据可知, 满足动力学模型拟合系数 $r_{2}>$ 0.99 , 标准误差 $s_{2}<0.2$ 的拟合结果见表中 “加粗斜 体” 的数据, 应用 Doyle 方程拟合数据没有一个模型 满足最概然机理拟合条件, 应用积分式 Coats-Red- fern 方程和微分式 Achar 方程拟合数据, 满足最概然 机理拟合条件也是模型 $\mathrm{A} 3$, 即过程机理 $g(x)=$ $[-\ln (1-\alpha)]^{1 / 3}$, 反应级数 $n=3$, 为集核和核生长机理. 由 Coats-Redfern方程计算所得的活化能为 19.97 $\mathrm{kJ} \cdot \mathrm{mol}^{-1}$, 与由 Achar 方程计算所得的活化能 18.55 $\mathrm{kJ} \cdot \mathrm{mol}^{-1}$ 相差不大, 两者平均 $19.26 \mathrm{~kJ} \cdot \mathrm{mol}^{-1}$. 这与文 献 ${ }^{18}$ 计算晶体生长激活能结果相近, 可认为晶化过 程和晶体生长过程同步进行. 物系非晶化和晶化过 程活化能较小, 对中间体分解过程应没有多大影响.

\section{3 中间体反应动力学机理}

3.3.1中间体生成反应

表 4 物系晶化过程反应动力学模型拟合系数 $r_{2}$ 及标准误差 $s_{2}$

Table4 Fitting coefficient $r_{2}$ and standard error $s_{2}$ of kinetic models of crystallization process

\begin{tabular}{|c|c|c|c|c|c|c|c|c|}
\hline \multirow{2}{*}{ Symbol } & \multicolumn{2}{|c|}{ Coats-Redfern equ. } & \multicolumn{2}{|c|}{ Doyle equ. } & \multicolumn{4}{|c|}{ Achar equ. } \\
\hline & $r_{2}$ & $\overline{s_{2}}$ & $r_{2}$ & $S_{2}$ & $r_{2}$ & $S_{2}$ & $E /\left(\mathrm{kJ} \cdot \mathrm{mol}^{-1}\right)$ & $\ln \left(A / \mathrm{s}^{-1}\right)$ \\
\hline D1 & 0.303 & 0.08 & 0.961 & 0.08 & 0.993 & 0.11 & 39.64 & 9.90 \\
\hline $\mathrm{D} 2$ & 0.592 & 0.09 & 0.961 & 0.09 & 0.993 & 0.08 & 33.50 & 7.14 \\
\hline D3 & 0.745 & 0.10 & 0.961 & 0.15 & 0.990 & 0.13 & 42.57 & 7.63 \\
\hline $\mathrm{R} 2$ & 0.830 & 0.05 & 0.961 & 0.05 & 0.997 & 0.03 & 25.25 & 5.53 \\
\hline R3 & 0.889 & 0.52 & 0.932 & 0.52 & 0.998 & 0.03 & 23.45 & 5.64 \\
\hline $\mathrm{A} 1$ & 0.502 & 0.06 & 0.961 & 0.06 & 0.996 & 0.05 & 28.85 & 7.52 \\
\hline A 1.5 & 0.928 & 0.04 & 0.960 & 0.04 & 0.994 & 0.04 & 23.70 & 6.45 \\
\hline A2 & 0.974 & 0.03 & 0.960 & 0.04 & 0.990 & 0.04 & 21.12 & 5.83 \\
\hline $\mathrm{A} 3$ & 0.991 & 0.02 & 0.960 & 0.03 & 0.998 & 0.06 & 18.55 & 5.09 \\
\hline P1 & 0.935 & 0.04 & 0.961 & 0.02 & 0.993 & 0.02 & 128.05 & 5.14 \\
\hline P2 & 0.991 & 0.02 & 0.961 & 0.01 & 0.976 & 0.06 & - & - \\
\hline P3 & 0.996 & 0.02 & 0.961 & 0.01 & 0.955 & 0.07 & - & - \\
\hline P4 & 0.997 & 0.02 & 0.961 & 0.01 & 0.935 & 0.08 & - & - \\
\hline $\mathrm{C} 1$ & 0.986 & 0.03 & 0.955 & 0.03 & 0.994 & 0.08 & 34.25 & 8.71 \\
\hline $\mathrm{C} 2$ & 0.816 & 0.05 & 0.965 & 0.05 & 0.992 & 0.10 & 39.64 & 9.90 \\
\hline
\end{tabular}


表 5 不同升温速率下中间体生成反应动力学模型拟合系数 $r_{3}$ 及标准误差 $s_{3}$

Table 5 Fitting coefficient $r_{3}$ and standard error $s_{3}$ of kinetic models of intermediate generation reaction at different heating rates

\begin{tabular}{|c|c|c|c|c|c|c|c|c|c|c|c|c|c|c|c|c|c|c|}
\hline \multirow{3}{*}{ Symbol } & \multicolumn{6}{|c|}{ Coats-Redfern equ. } & \multicolumn{6}{|c|}{ Doyle equ. } & \multicolumn{6}{|c|}{ Achar equ. } \\
\hline & \multicolumn{2}{|c|}{$5^{\circ} \mathrm{C} \cdot \mathrm{min}^{-1}$} & \multicolumn{2}{|c|}{$10^{\circ} \mathrm{C} \cdot \mathrm{min}^{-1}$} & \multicolumn{2}{|c|}{$15^{\circ} \mathrm{C} \cdot \mathrm{min}^{-1}$} & \multicolumn{2}{|c|}{$5^{\circ} \mathrm{C} \cdot \min ^{-1}$} & \multicolumn{2}{|c|}{$10^{\circ} \mathrm{C} \cdot \mathrm{min}^{-1}$} & \multicolumn{2}{|c|}{$15^{\circ} \mathrm{C} \cdot \mathrm{min}^{-1}$} & \multicolumn{2}{|c|}{$5^{\circ} \mathrm{C} \cdot \min ^{-1}$} & \multicolumn{2}{|c|}{$10^{\circ} \mathrm{C} \cdot \mathrm{min}^{-1}$} & \multicolumn{2}{|c|}{$15^{\circ} \mathrm{C} \cdot \min ^{-1}$} \\
\hline & $r_{3}$ & $S_{3}$ & $r_{3}$ & $S_{3}$ & $r_{3}$ & $S_{3}$ & $r_{3}$ & $s_{3}$ & $r_{3}$ & $S_{3}$ & $r_{3}$ & $S_{3}$ & $r_{3}$ & $s_{3}$ & $r_{3}$ & $s_{3}$ & $r_{3}$ & $s_{3}$ \\
\hline D1 & 0.881 & 0.46 & 0.866 & 0.52 & 0.855 & 0.52 & 0.910 & 0.37 & 0.896 & 0.51 & 0.889 & 0.51 & 0.996 & 0.11 & $0.98 /$ & 0.23 & 0.994 & 0.21 \\
\hline $\mathrm{D} 2$ & 0.906 & 0.48 & 0.896 & 0.53 & 0.886 & 0.53 & 0.927 & 0.47 & 9.17 & 0.53 & 0.909 & 0.53 & 0.965 & 0.21 & 0.917 & 0.26 & 0.965 & 0.25 \\
\hline D3 & 0.931 & 0.47 & 0.929 & 0.53 & 0.920 & 0.53 & 0.945 & 0.07 & 0.942 & 0.07 & 0.935 & 0.06 & 0.991 & 0.11 & 0.987 & 0.21 & 0.990 & 0.23 \\
\hline $\mathrm{R} 2$ & 0.896 & 0.23 & 0.891 & 0.26 & 0.881 & 0.27 & 0.937 & 0.23 & 0.931 & 0.26 & 0.925 & 0.65 & 0.993 & 0.03 & 0.966 & 0.04 & 0.995 & 0.05 \\
\hline R3 & 0.983 & 0.51 & 0.833 & 0.51 & 0.835 & 0.52 & 0.867 & 0.51 & 0.873 & 0.52 & 0.876 & 0.31 & 0.994 & 0.04 & 0.981 & 0.06 & 0.995 & 0.06 \\
\hline A1 & 0.938 & 0.23 & 0.939 & 0.23 & 0.923 & 0.24 & 0.959 & 0.22 & 0.960 & 0.24 & 0.957 & 0.36 & 0.995 & 0.06 & 0.987 & 0.10 & 0.995 & 0.06 \\
\hline A 1.5 & 0.921 & 0.15 & 0.921 & 0.15 & 0.918 & 0.18 & 0.959 & 0.15 & 0.960 & 0.17 & 0.956 & 0.17 & 0.950 & 0.13 & 0.893 & 0.16 & 0.972 & 0.16 \\
\hline A2 & 0.897 & 0.11 & 0.811 & 0.80 & 0.898 & 0.13 & 0.959 & 0.11 & 0.960 & 0.12 & 0.957 & 0.13 & 0.850 & 0.38 & 0.670 & 0.20 & 0.942 & 0.19 \\
\hline A3 & 0.811 & 0.80 & 0.837 & 0.23 & 0.832 & 0.27 & 0.959 & 0.07 & 0.960 & 0.08 & 0.957 & 0.65 & 0.585 & 0.21 & 0.182 & 0.24 & 0.838 & 0.23 \\
\hline P1 & 0.837 & 0.23 & 0.667 & 0.12 & 0.822 & 0.34 & 0.910 & 0.23 & 0.896 & 0.25 & 0.889 & 0.26 & 0.703 & 0.37 & 0.999 & 0.01 & 0.999 & 0.03 \\
\hline P2 & 0.667 & 0.12 & 0.271 & 0.08 & 0.623 & 0.13 & 0.910 & 0.11 & 0.896 & 0.12 & 0.889 & 0.32 & 0.877 & 0.13 & 0.975 & 0.11 & 0.695 & 0.13 \\
\hline P3 & 0.271 & 0.08 & 0.297 & 0.06 & 0.283 & 0.09 & 0.910 & 0.07 & 0.896 & 0.08 & 0.889 & 0.12 & 0.888 & 0.39 & 0.967 & 0.21 & 0.773 & 0.58 \\
\hline P4 & 0.297 & 0.06 & 0.398 & 0.32 & 0.215 & 0.07 & 0.910 & 0.05 & 0.896 & 0.06 & 0.889 & 0.03 & 0.891 & 0.18 & 0.960 & 0.18 & 0.792 & 0.19 \\
\hline $\mathrm{C} 1$ & 0.993 & 0.02 & 0.993 & 0.02 & 0.986 & 0.05 & 0.997 & 0.02 & 0.991 & 0.06 & 0.993 & 0.05 & 0.996 & 0.08 & 0.990 & 0.15 & 0.994 & 0.14 \\
\hline $\mathrm{C} 2$ & 0.996 & 0.04 & 0.996 & 0.04 & 0.990 & 0.10 & 0.997 & 0.05 & 0.991 & 0.11 & 0.993 & 0.10 & 0.996 & 0.11 & 0.991 & 0.20 & 0.994 & 0.18 \\
\hline
\end{tabular}

表 5 为在不同的升温速率下, 用 Coats-Redfern 方程(式(3))、Doyle 方程(式(4)) 和 Achar-BrindleySlarp-Wendworth 方程(式(5))对图 6 中第 2 个 DTG 峰 数据进行拟合, 得到的中间体生成反应固相反应动 力学模型拟合的拟合系数及误差. 最概然动力学模 型要求拟合系数 $r_{3}>0.99$, 标准误差 $s_{3}<0.2$, 并且不同 的升温速率同为一个反应动力学模型.

由表 5 可知, 只有 $\mathrm{C} 1$ 和 $\mathrm{C} 2$ 机理函数(相界面反 应幂律机理模型)符合的机理函数线性拟合的要求, 由相应方程计算所得的活化能见表 6. 与由 Ozawa 法、Kissinger法计算得到的活化能比较, $\mathrm{C} 2$ 机理的 活化能与由 Ozawa 法和 Kissinger 法计算得到的活 化能比较接近, 可认为 $\mathrm{LiCoPO}_{4}$ 生成反应机理函数
为 $g(x)=(1-\alpha)^{-1}$.

\subsection{2 中间体分解反应机理}

采用以上同样的方法, 对中间体的热分解过程 进行分析, 中间体热分解遵循 Doyle 方程中的相界 面反应幂律机理模型 $\mathrm{C} 2$, 三个不同升温度下热反应 的平均表观活化能为 $54.2 \mathrm{~kJ} \cdot \mathrm{mo}^{-1}$, 与由 Ozawa法、 Kissinger 法计算得到的活化能比较相差较大, 可能 是因为 Ozawa 法、Kissinger 法计算中认为活化能与 机理无法的假设, 与事实不相称, 如果从中间体的 “ $\mathrm{CoHPO}_{4} \cdot \mathrm{LiCoPO}_{4} \cdot \mathrm{Co}_{2}(\mathrm{OH}) \mathrm{PO}_{4} \cdot \mathrm{Li}_{3} \mathrm{PO}_{4}$ ” 组成初步 分析, 过程为 $\mathrm{CoHPO}_{4}$ 和 $\mathrm{Co}_{2}(\mathrm{OH}) \mathrm{PO}_{4}$ 间脱水反应活 化能不会太大, 从图 8 中 $303.1{ }^{\circ} \mathrm{C}$ 处的吸热很少也 得到证明, 故中间体的热分解反应平均表观活化能

表 6 不同升温速率下中间体生成反应动力学模型活化能及指前因子

Table 6 Activation energies and exponential factors of kinetic models of intermediate generation reaction at different heating rates

\begin{tabular}{|c|c|c|c|c|c|}
\hline \multirow{2}{*}{$\beta /\left({ }^{\circ} \mathrm{C} \cdot \min ^{-1}\right)$} & \multirow{2}{*}{ Kinetics equ. } & \multicolumn{2}{|c|}{$\mathrm{C} 1$} & \multicolumn{2}{|c|}{$\mathrm{C} 2$} \\
\hline & & $E /\left(\mathrm{kJ} \cdot \mathrm{mol}^{-1}\right)$ & $\ln \left(A / \mathrm{s}^{-1}\right)$ & $E /\left(\mathrm{kJ} \cdot \mathrm{mol}^{-1}\right)$ & $\ln \left(A / \mathrm{s}^{-1}\right)$ \\
\hline \multirow[t]{3}{*}{5} & Coats-Red & 10.59 & 0.29 & 29.75 & 5.51 \\
\hline & Doyle & 19.16 & 4.93 & 36.43 & 9.33 \\
\hline & Achar & 77.75 & 17.30 & 58.46 & 12.35 \\
\hline \multirow[t]{3}{*}{10} & Coats-Red & 18.52 & 2.59 & 47.88 & 12.67 \\
\hline & Doyle & 27.37 & 6.43 & 52.03 & 12.68 \\
\hline & Achar & 93.45 & 20.20 & 66.12 & 13.31 \\
\hline \multirow[t]{3}{*}{15} & Coats-Red & 18.38 & 0.57 & 47.75 & 10.44 \\
\hline & Doyle & 26.01 & 6.80 & 52.02 & 12.90 \\
\hline & Achar & 116.20 & 29.47 & 90.02 & 22.94 \\
\hline
\end{tabular}


取值为 $54.2 \mathrm{~kJ} \cdot \mathrm{mol}^{-1}$ 相对合理, 认为中间体热分解 与机理选择有关, 中间体容易生成, 但也容易分解.

\section{4 结 论}

以氢氧化锂、磷酸二氢铵和醋酸钴为原料, 采 用前驱体固相活化法在空气气氛中制备 $\mathrm{LiCoPO}_{4}$ 过 程中, 含 $\mathrm{Li}^{+}$前驱体在 $210-500{ }^{\circ} \mathrm{C}$ 发生脱水脱氨反 应时, 因为反应生成的 $\mathrm{CoHPO}_{4}$ 和 $\mathrm{Co}_{2}(\mathrm{OH}) \mathrm{PO}_{4}$ 不能 及时接触并进一步反应, 存在一个酸碱共存的中间 体 $\left(\mathrm{CoHPO}_{4} \cdot \mathrm{LiCoPO}_{4} \cdot \mathrm{Co}_{2}(\mathrm{OH}) \mathrm{PO}_{4} \cdot \mathrm{Li}_{3} \mathrm{PO}_{4}\right)$.

\section{(I) $\mathrm{NH}_{4} \mathrm{CoPO}_{4} \cdot \mathrm{H}_{2} \mathrm{O} \rightarrow \mathrm{NH}_{4} \mathrm{CoPO}_{4}+\mathrm{H}_{2} \mathrm{O}$ \\ (II) $\mathrm{NH}_{4} \mathrm{CoPO}_{4} \cdot \mathrm{H}_{2} \mathrm{O}+\mathrm{LiOH}+\mathrm{Li}_{3} \mathrm{PO}_{4} \rightarrow$ intermediate $+\mathrm{NH}_{3}+\mathrm{H}_{2} \mathrm{O}$ \\ (III) intermediate $\rightarrow \mathrm{LiCoPO}_{4}+\mathrm{H}_{2} \mathrm{O}$}

气氛影响中间体的存在, 中间体在空气中比在 氮气中容易形成, 且其组成与反应投料量有关, 物 系晶化和非晶化过程对中间体的形成和分解影响 不大. 在空气中, 中间体的生成和热分解反应过程 均遵循界面反应幂律机理, 活化能分别为 50.0 和 $54.2 \mathrm{~kJ} \cdot \mathrm{mol}^{-1}$.

\section{References}

(1) Zheng, J. C.; Li, X. H.; Wang, Z. X.; Li, J. H.; Wu, L.; Li, L. J.; Guo, H. J. Acta Phys. -Chim. Sin. 2009, 25, 1916. [郑俊超, 李 新海, 王志兴, 李金辉, 伍 凌, 李灵均, 郭华军. 物理化学学 报. 2009, 25, 1916.]

(2) Wolfenstine, J.; Allen, J. J. Power Sources 2004, 136, 150.

(3) Zhou, F.; Cococcioni, M.; Kang, K.; Ceder, G. Electrochem. Commun. 2004, 6, 1144.

(4) Rissouli, K.; Benkhouja, K.; Ramos-Barrado, J. R.; Julien, C. Mater. Sci. Eng. B 2003, 98, 185.

(5) Okada, S.; Ueno, M.; Uebou, Y.; Yamaki, J. I. J. Power Sources 2005, 146, 565 .

(6) Goni, A.; Lezama, L.; Barberis, G. E.; Pizarro, J. L.; Arriortua, M. I.; Rojo, T. J. Magn. Magn. Mater. 1996, 164, 251.

(7) Brown, P. J.; Frsyth, J. B.; Tasset, F. Solid-State Sci. 2005, 7, 682.

(8) Santoro, R. P.; Segal, D. J.; Newnham, R. E. J. Phys. Chem.
Solids 1966, 27, 119.

(9) Van Aken, B. B.; Rivera, J. P.; Schmid, H.; Fiebig, M. F. Nature 2007, 449, 702 .

(10) Ehrenberg, H.; Bramnik, N. N.; Senyshyn, A.; Fuess, H. Solid State Sci. 2009, 11, 18.

(11) Bramnik, N. N.; Bramnik, K. G.; Baehtz, C.; Ehrenberg, H. J. Power Sources 2005, 145, 74.

(12) Wolfenstine, J.; Poese, B.; Allen, J. L. J. Power Sources 2004, $138,281$.

(13) Wolfenstine, J.; Read, J.; Allen, J. L. J. Power Sources 2007, 163,1070 .

(14) Wolfenstine, J.; Lee, U.; Poese, B.; Allen, J. L. J. Power Sources 2005, 144, 226.

(15) Grigorova, V.; Roussev, D.; Deniard, P.; Jobic, S. J. Phys. Chem. Solids 2005, 66, 1598.

(16) Deniard, P.; Dulac, A. M.; Roequefdte, X.; Grigorova, V.; Lebacq, O.; Pasturel, A.; Jobic, S. J. Phys. Chem. Solids 2004, $65,229$.

(17) Han, D. W.; Kang, Y. M.; Yin, R. Z.; Song, M. S.; Kwon, H. S. Electrochem. Commun. 2009, 11, 137.

(18) Huang, Y. H.; Tong, Z. F.; Lan, J. J.; Chen, Y. Z. J. Yunnan University (Natural Science) 2010, 32, 314. [黄映恒, 童张法, 蓝建京, 陈义族. 云南大学学报: 自然科学版, 2010, 32,314.]

(19) Huang, Y. H.; Tong, Z. F.; Lan, J. J.; Chen, Y. Z. The Chinese Journal of Process Engineering 2010, 10, 179. [黄映恒, 童张 法, 蓝建京, 陈义族. 过程工程学报, 2010, 10, 179.]

(20) Huang, Y. H.; Tong, Z. F.; Liao, S.; Lan, J. J.; Chen, Y. Z. Journal of Chemical Engineering of Chinese Universities 2010, 24, 967. [黄映恒, 童张法, 廖 森, 蓝建京, 陈义族. 高校化学 工程学报, 2010, 24, 967.]

(21) Koleva, G. V. Spectrochimica Acta Part A: Molecular and Biomolecular Spectroscopy 2005, 62, 1196.

(22) Koleva, G. V. Spectrochimica Acta Part A: Molecular and Biomolecular Spectroscopy, 2007, 66, 413.

(23) Ruan, Y. L.; Tang, Z. Y. Acta Phys. -Chim. Sin. 2008, 24, 873. [阮艳莉, 唐致远. 物理化学学报, 2008, 24, 873.]

(24) Conesa, J. A.; Marcilla, A.; Caballero, J. A.; Font, R. J. Anal. Appl. Pyrolysis 2001, 58-59, 617.

(25) Ozawa, T. J. Therm. Anal. 1970, 2, 301.

(26) Kissinger, H. E. J. Anal. Chem. 1957, 29, 1702.

(27) Sestak, J.; Berggren, G. Thermochim. Acta 1971, 3, 1. 